\title{
Schlieren imaging: a powerful tool for atmospheric plasma diagnostic
}

\author{
Enrico Traldi ${ }^{1}$, Marco Boselli ${ }^{1,2}$, Emanuele Simoncelli ${ }^{1}$, Augusto Stancampiano ${ }^{3}$, Matteo Gherardi ${ }^{1,2}$, \\ Vittorio Colombo ${ }^{1,2^{*}}$ and Gary S. Settles ${ }^{4^{*}}$
}

\footnotetext{
* Correspondence: vittorio. colombo@unibo.it; gss2@psu.edu ${ }^{1}$ Department of Industrial Engineering, Alma Mater Studiorum - Università di Bologna, Viale del Risorgimento 2, 40136 Bologna, Italy ${ }^{4}$ FloViz Inc., Port Matilda, PA 16870, USA

Full list of author information is available at the end of the article
}

\begin{abstract}
Schlieren imaging has been widely used in science and technology to investigate phenomena occurring in transparent media. In particular, it has proven to be a powerful tool in fundamental studies and process optimization for atmospheric pressure plasma diagnostics, providing qualitative and (in some cases) also quantitative information on the fluid-dynamic characteristics of plasmas generated by many different types of sources. However, obtaining significant and reliable results by schlieren imaging can be challenging, especially when considering the variety of geometries and applications of atmospheric pressure plasma sources. Therefore, it is necessary to adopt solutions that can address the specific issues of different plasma-assisted processes. In this paper, an overview on the use of the schlieren imaging technique for atmospheric pressure plasma characterization is presented. In the first part, the physical principles behind this technique and the different setups that can be adopted to perform it are presented. In the second part, examples of schlieren imaging applied to different kinds of atmospheric pressure plasmas (non-equilibrium plasma jets, plasma actuators for flow control and thermal plasma sources) are presented, showing how it was used to characterize the fluid-dynamic behavior of plasma-assisted processes and reporting best practices in performing this diagnostic technique.
\end{abstract}

Keywords: Schlieren imaging, Atmospheric pressure plasma, Plasma diagnostics, Best practices

\section{Introduction}

The number of industrial and biomedical applications of plasmas at atmospheric pressure has been steadily increasing over the past decade [1, 2]: nowadays, plasma applications include materials treatment for the activation of surfaces or coating deposition, ozone synthesis, fuel conversion and the treatment of liquids $[3,4]$ bacterial inactivation and plasma-assisted medical therapies [5], plasma actuators for active flow control on airfoils, gas turbine blades and wind turbines [6, 7], and high temperature material processing, such as plasma spraying, arc cutting and arc welding [8-10], etc. This versatility is gained by several possible combinations of driving power supply, management and composition of working gas, formation and propagation of the discharge and source architecture.

The need for an optimization of plasma assisted processes has prompted the scientific community to thoroughly investigate the physics of plasmas and their complex behavior

(c) The Author(s); licensee Springer on behalf of EPJ. 2018 Open Access This article is distributed under the terms of the Creative Commons Attribution 4.0 International License (http://creativecommons.org/licenses/by/4.0/), which permits unrestricted use, distribution, and reproduction in any medium, provided you give appropriate credit to the original author(s) and the source, provide a link to the Creative Commons license, and indicate if changes were made. 
in interacting with various materials (e.g. biological materials, liquids, etc.) by means of a range of diagnostic techniques [11-13]; among these, a prominent role is held by schlieren imaging, which is nowadays widespread and extensively used for the investigation of plasma processes by studying phenomena occurring in transparent media [14].

In fact, nearly all plasma processes have in common the fact that plasma is generated and propagates in a fluid medium. The fluid-dynamics inside and outside the plasma region play a critical role in determining plasma chemical composition and discharge morphology, the propagation of reactive species, interaction and mixing of plasma with the surrounding atmosphere, residence time of precursors in the plasma region and resulting deposition rates, among other process characteristics. From this perspective it is easy to understand why schlieren imaging, which allows one to see "the invisible" [14], is considered a powerful tool for characterizing atmospheric pressure plasma processes and why its use is steadily increasing.

Against this background, this paper is meant as an introduction to schlieren imaging and its use as a tool for plasma diagnostics, highlighting its potentialities and providing the readers with practical examples associated with different atmospheric pressure plasma processes. The expected audience includes plasma scientists that are current and potential users of this technique. The paper is developed along two main parts: a theoretical part, concerning the physical fundamentals of the technique, the many instruments used to implement it and their preferred configurations, and a more practical part meant to deal with several examples of its use for the study of the behavior of different plasma sources and processes, describing both the type and quality of the obtainable information and the setups and practical measures adopted. As a matter of fact, even focusing only on atmospheric pressure plasmas, the temperature ranges from room temperature to several thousand $\mathrm{K}$, while power can go from a few $\mathrm{W}$ to several $\mathrm{kW}$; the characteristics of the adopted schlieren setup should be accordingly tuned. To show how this tuning of schlieren optics is performed to achieve optimal results, a selection of best practices in the conduct of experimental activities to investigate different kinds of processes assisted by atmospheric pressure plasmas is also reported in the second part of this paper, similarly to a previous paper from some of the authors concerning best practices in ICCD diagnostics of plasmas [15].

\section{Schlieren optical principles and instrument design}

Schlieren optics arose in the seventeenth century along with microscopes and telescopes, but unlike those instruments, schlieren required two more centuries for science to mature enough to have useful applications for it [16]. Since the mid-nineteenth century schlieren optics have found use in all branches of science where transparent phenomena require visualization, including phenomena in solids, liquids, gases, and plasmas. Inventor August Toepler [17] deliberately gave the instrument a catchy name: the method of streaks (Schlieren in German).

Fundamentally, schlieren optics are able to detect changes in the refractive index $\mathrm{n}$ of a medium through which a light beam is passed. If the beam travels in the z-direction, then refractive-index gradients $\partial \mathrm{n} / \partial \mathrm{x}$ and $\partial \mathrm{n} / \partial \mathrm{y}$ refract light rays, but this is normally not visible to the eye. In schlieren optics the light beam is brought to a sharp focus and the refracted rays are detected by applying a spatial filter. 
This is illustrated in Fig. 1a, a diagram of a lens-type schlieren system similar to Toepler's original instrument. A small bright light source LS provides illumination, propagating in the $\mathrm{z}$-direction, that is collimated by lens L1 and focused by lens L2. Between the lenses lies a "schlieren object" S represented by squiggly lines. Positive and negative $n$-gradients $\partial \mathrm{n} / \partial \mathrm{y}$ inside the schlieren object refract light rays upward (dashed line) and downward (solid line), respectively. (The angles of refraction are exaggerated in Fig. 1a for clarity, and are typically so small that they are measured in arcseconds, as in astronomy.)

The detection step occurs at the beam focus in Fig. 1a, where a sharp opaque edge (razor blade or knife-edge KE) blocks about half of the light beam. The upward-refracted ray is seen to miss the focus and the knife edge, so it proceeds to illuminate a point on the sensor of camera $C$. The downward-refracted ray is blocked by the knife edge, producing a corresponding dark point on the camera sensor. Generalizing to all the rays in the light beam, a schlieren image is formed in the camera, where the features of $S$ are represented by light and dark zones against a uniform grey background.

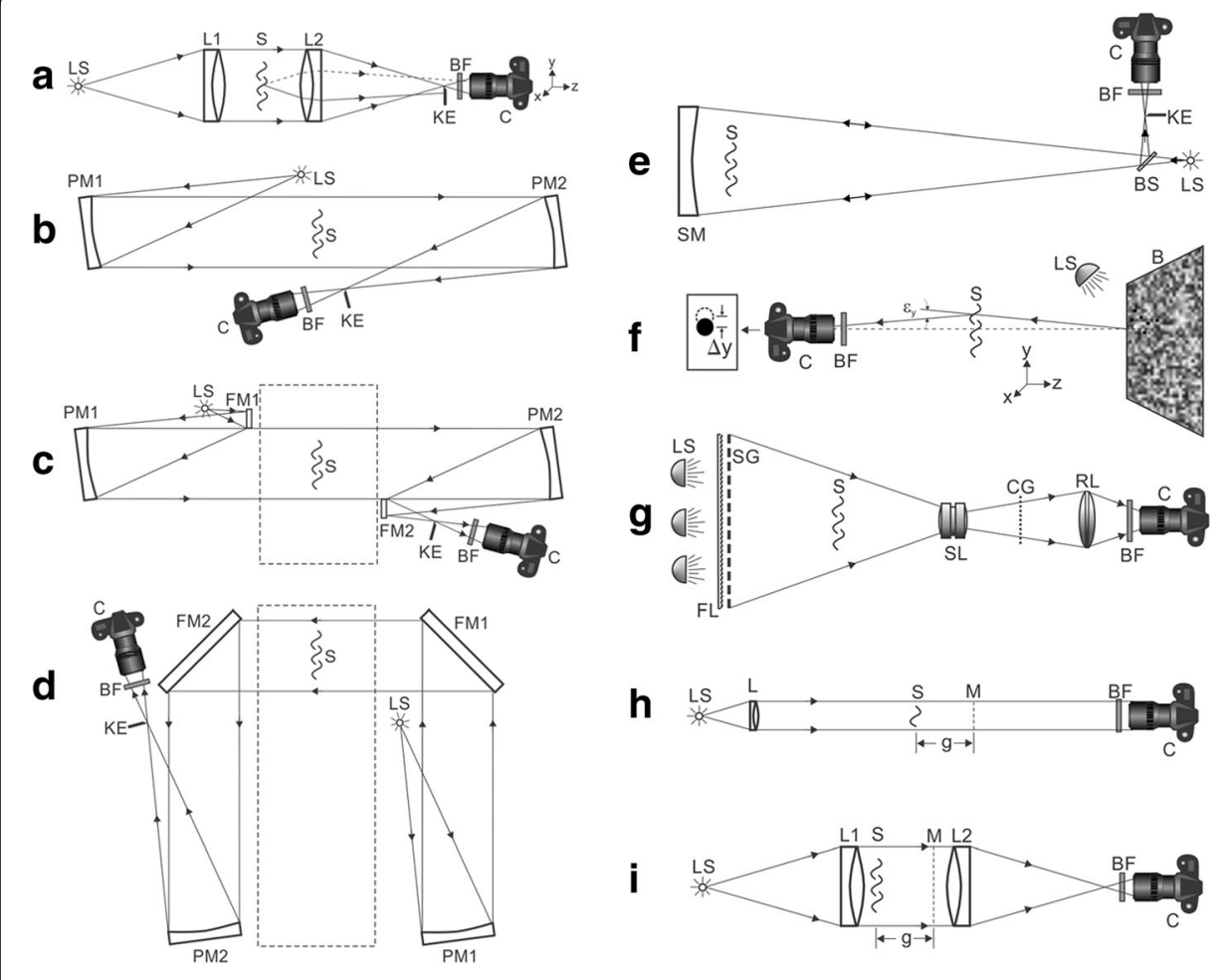

Fig. 1 Diagrams of different schlieren instruments: Toepler's lens-type system (a), simple "z-type" system (b) folded z-type systems with both non-parallel schlieren beams folded (c) and with the parallel beam folded twice (d), single-mirror, double-pass schlieren arrangement (e), Background-Oriented Schlieren (BOS) (f), focusing (lens-and-grid) schlieren system (g), direct shadowgraph system (h) and "focused shadowgraph" system (i). The individual components of these setups are described in detail in the text. List of the used abbreviations: B - background; BF - bandpass filter; BS - beamsplitter; C- camera; CG - cutoff grid; FL Fresnel lens; FM1 - folding mirror 1; FM2 - folding mirror 2; $\mathrm{g}$ - defocusing distance; KE - knife-edge; L - lens; LS - light source; L1 -schlieren field lens 1; L2 - schlieren field lens 2; M - plane of camera focus; PM1 - parabolic mirror 1; PM2 - parabolic mirror 2; RL - relay lens; S - schlieren object (plasma); SG source grid; SL - schlieren lens; SM - spherical mirror; $x, y, z$ - Cartesian coordinates; $\Delta y$ - ray displacement in $y$-direction; $\varepsilon_{y}$ - refraction angle in $y$-direction 
Twentieth-century schlieren pioneer Schardin [18] recognized that the schlieren instrument actually performs two simultaneous functions: 1) it images the schlieren object (and any associated opaque objects) sharply on the camera sensor, and 2) it discriminates refracted from un-refracted rays by way of the knife-edge, thus rendering a greyscale amplitude image of $\mathrm{S}$, which is itself a pure phase disturbance. Thus schlieren optics make visible the otherwise-invisible.

A more sophisticated optical description, beyond the present scope, treats Fig. 1a as a Fourier Optical Processor ([16], App. B), and the knife-edge as a half-plane cutoff of the resulting spatial frequency spectrum. Many other choices are available for spatial filters in the knife-edge plane, including some that produce schlieren images in color $[16,18,19]$.

\section{Schlieren theory}

Detailed mathematical analyses of the schlieren technique are available from many sources, e.g. [16, 18, 20-25]. For present purposes, it is sufficient to outline the geometric-optics approach by which the properties of schlieren object $\mathrm{S}$ are related to its image as captured by the camera. Wave-optics, especially diffraction effects, also play a role in some circumstances that are beyond the present scope.

Schlieren optics are integrative. In other words, the effects of refractive-index gradients are integrated along the path of a light ray in the z-direction. The resulting angle of refraction of a light ray in the y-direction, $\varepsilon_{y}$, due to a refractive-index gradient $\partial \mathrm{n} / \partial \mathrm{y}$ in schlieren object $\mathrm{S}$ can be written as:

$$
\varepsilon_{y}=\frac{1}{n} \int \frac{\partial n}{\partial y} d z
$$

For the simplest case of a planar 2D schlieren object where $\mathrm{n}$ may vary in $\mathrm{x}$ and $\mathrm{y}$ but not $\mathrm{z}$, simplifying assumptions allow this integral to be evaluated as:

$$
\varepsilon_{y}=\frac{Z}{n_{\infty}} \frac{\partial n}{\partial y}
$$

Where $\mathrm{n}_{\infty}$ is the refractive index of the surrounding medium and $\mathrm{Z}$ is the thickness of the schlieren object in the z-direction. This equation shows that the refraction angle $\varepsilon_{y}$ is proportional to the strength of the gradient $\partial \mathrm{n} / \partial \mathrm{y}$. For every schlieren system there is some threshold value of $\partial \mathrm{n} / \partial \mathrm{y}$ below which $\varepsilon_{y}$ becomes too small to be observed or measured.

Applying trigonometry and the characteristics of the schlieren optics, $\varepsilon_{\mathrm{y}}$ can also be expressed as:

$$
\varepsilon_{y}=\frac{a}{f_{2}}
$$

Where $\mathrm{f}_{2}$ is the focal length of lens L2 in Fig. 1a and $a$ is the distance that a refracted light ray is displaced above or below the beam focus in the knife-edge plane. This distance can be measured by various photometric or colorimetric methods, e.g. [26], yielding the distribution of $\partial \mathrm{n} / \partial \mathrm{y}$ in the schlieren object from information in the schlieren image and Eqs. 2 and 3 above. The integration of $\partial \mathrm{n} / \partial \mathrm{y}$, starting from a 
known point of $n_{\infty}$ in the image, yields a profile of refractive index $n$ in the schlieren object.

Once $\mathrm{n}$ is found from a schlieren image, it can be related to gas density $\rho$ by the Gladstone-Dale equation [16, 20],

$$
n=k \rho+1
$$

where the Gladstone-Dale constant $k=2.33 \times 10^{-4} \mathrm{~m}^{3} / \mathrm{kg}$ for air with illumination at $0.3562 \mu \mathrm{m}$ wavelength, for example. For plasmas the Gladstone-Dale relationship is more complicated (see for instance Merzkirch [20] Ch. 3 and Vasiliev [24] Sec. 44) and can be expressed as,

$$
n=\frac{1}{4 \pi \varepsilon_{0}} 2 \alpha_{+}(\lambda) \rho_{+}+\frac{1}{4 \pi \varepsilon_{0}} 2 \alpha_{n}(\lambda) \rho_{n}-\frac{1}{4 \pi \varepsilon_{0}} \frac{e^{2} \lambda^{2}}{2 \pi m_{e} c_{0}^{2}} \rho_{e}+1
$$

where $\varepsilon_{0}$ is the free space permittivity $\alpha_{+}(\lambda)$ and $\alpha_{n}(\lambda)$ are the polarizability of ions and neutrals, respectively, dependent on the light wavelength $\lambda, \rho_{+}$and $\rho_{n}$ are respectively the densities of ions and neutrals, $e$ is the electronic charge, $m_{e}$ is the electron mass, $c_{0}$ is the light velocity and $\rho_{e}$ is the density of electrons [27]. The contribution of excited species can usually be neglected, as resonance effects can alter refraction index, but the required wavelengths are usually in the vacuum ultraviolet region for common plasma gases; light sources used in schlieren imaging, even lasers, usually emit in the visible light wave range, so they won't be affected by excited species [28].

Finally, a state equation can be used to relate $\rho$ to temperature T and pressure $\mathrm{p}$, usually the perfect gas equation of state:

$$
p=\rho R T
$$

where $\mathrm{R}$ is the gas constant of the particular gas in use. Some plasmas also follow perfect-gas behavior approximately [29], but state equations for various types of plasma have been the subject of research studies for decades. With high electron temperature (more than $10 \mathrm{k} \mathrm{K}$ for argon, oxygen or nitrogen plasmas in Local Thermodynamic Equilibrium at atmospheric pressure) dissociation and ionization further decrease the gas density. For instance, the gas constant $\mathrm{R}$ at $20 \mathrm{k} \mathrm{K}$ for argon is 2 times the room temperature one, while for oxygen and nitrogen is respectively 3 and 4 times higher. Non equilibrium conditions further increase this effect [30].

Equation 5 reveals that, at constant atmospheric pressure p, $\rho$ and $\mathrm{T}$ are inversely related. Thus, temperature differences in the air lead to refractive-index gradients that show up in a schlieren image, allowing the schlieren technique to visualize convective heat transfer. Two other phenomena that schlieren can image are the mixing of different media such as plasma and gas, and compressible flows including shock waves.

Many schlieren objects do not have the planar 2D symmetry invoked above, but do have radial symmetry about an axis. This class includes gas and plasma jets from round orifices and many supersonic flows, for example. When the schlieren beam passes through such flows perpendicular to their axes, the effects of n-gradients are pathaveraged to yield the schlieren image. One may apply the inverse Abel transformation [31] to such path-averaged results in order to recover the true density profile. An example is given in Ref. [32]. 
When no symmetry is present and the schlieren object is fully $3 \mathrm{D}$, quantitative analysis of single schlieren images as described above is no longer possible. Instead, a tomographic approach yielding many images from different angles is required. An example of such an ambitious experiment is given in Ref. [33].

\section{Types of Schlieren instruments}

Toepler's lens-type schlieren system, Fig. 1a, is one of the setups that has been used to study plasmas, but it is seldom used when the required field-of-view is larger than about $150 \mathrm{~mm}$. The reason is that, already at this diameter, lenses of sufficient quality are quite expensive. Achromatic objective lenses for refractor telescopes have the quality and long focal lengths necessary for schlieren instruments, but inexpensive single-element lenses do not (not even when monochromatic illumination is used).

For diameters of $100 \mathrm{~mm}$ to $1 \mathrm{~m}$ or more, spherical or parabolic telescope mirrors are routinely used instead of lenses in schlieren instruments. Having only one precise optical surface rather than several, and not requiring high internal optical quality, mirrors in this size range are much less expensive than lenses would be if they were available.

Figure $1 \mathrm{~b}$ is a diagram of a "z-type" schlieren instrument using two first-surface parabolic mirrors, PM1 and PM2, as field elements in place of lenses. This has been the most common type of instrument used in studying plasma phenomena to date. Its name comes from the similarity of its layout to the letter $z$. Aside from the folding of the schlieren beam due to mirror reflections, it operates very much like the in-line lens system of Fig. 1a.

Since the mirrors are figured for on-axis use but are used off-axis in Fig. 1b, certain optical aberrations arise that can cause uneven illumination of the schlieren image even when $\mathrm{S}$ is not present. To minimize these, one must tilt the parabolic mirrors toward opposite sides of the parallel schlieren beam and also minimize the tilt angles of the mirrors by choosing mirrors with long focal lengths, preferable at least $f / 8$ if not f/10. More detail on this issue is found in Refs. [16, 22].

Sometimes the long focal lengths in Fig. 1b make the schlieren system unwieldy in cramped quarters, requiring the entering and leaving light beams to be folded again as shown in Fig. 1c. In this case planar first-surface folding mirrors FM1 and FM2 are used. These cause no additional optical aberrations if the tilt angles of PM1 and PM2 are not increased, but they do lead to a more-complicated optical alignment procedure.

The folded z-type schlieren system is especially useful for looking through the windows of a test facility such as a wind tunnel (indicated by a dashed outline in Fig. 1c, d). Wind tunnels tend to be housed in long narrow rooms, the narrow dimension necessitating the folding of schlieren optics. In extreme cases the parallel schlieren beam can be folded twice, as shown in Fig. 1d. Such a compact schlieren system serves the transonic wind tunnel of DLR Göttingen, for example, where it is entirely inside the pressure plenum of the facility. Note that the large first-surface folding mirrors FM1 and FM2 must be flat to better than $1 / 4$ wavelength of light across their surfaces. They may cost as much or more than the parabolic mirrors.

Because of the double folding, the parabolic mirrors in Fig. 1d appear to both be tilted in the same direction, but they are actually tilted toward opposite sides of the 
parallel schlieren beam. There is a tendency to tilt PM1 to the other side, making a schlieren system of " $\Omega$-type." This is unwise, though, because it doubles rather than cancels the astigmatic aberration of the schlieren system [16, 22].

One more mirror-based schlieren instrument of practical interest is shown in Fig. 1e. A spherical mirror SM, when illuminated by a small light source LS at its radius of curvature, returns the light exactly to the source. A beam-splitter BS deflects half of the reflected beam to the side, where knife-edge $\mathrm{KE}$ is inserted at the focus and camera $\mathrm{C}$ records a schlieren image. The schlieren object $\mathrm{S}$ is located close to the mirror, and (unlike Fig. 1a-d) is illuminated by non-parallel light. This is called a single-mirror, double-pass schlieren arrangement because, with proper alignment, an illuminating ray passes twice through each point in $S$ and thus receives twice the deflection angle of the single-pass instruments described earlier. This plus the large distance between LS and SM (twice the focal length of SM) make this schlieren system very sensitive to weak refractive disturbances. The mirror can be quite large; a double-pass system with a mirror of $1 \mathrm{~m}$ diameter is described in Ch. 4 of [16]. If a parabolic mirror is used in place of SM, then a working schlieren instrument can still be made with the addition of some correcting optics [34].

Figure 1f diagrams a relatively-new synthetic schlieren method called BackgroundOriented Schlieren (BOS). Camera C images a distant patterned background screen B. Images of schlieren object $\mathrm{S}$ in front of $\mathrm{B}$ contain small shifts $\Delta \mathrm{y}$ in background structure due to refraction. There is no knife-edge in this simple synthetic schlieren instrument, but digital image processing is able to measure $\Delta y$ over the entire image and construct a pseudo-schlieren image without the lenses or mirrors of Fig. 1a-e.

BOS has attracted a lot of interest, but has apparently been used only once to date for imaging plasmas [35]. It has great potential for large-scale and outdoor schlieren imaging with natural backgrounds, but also suffers from low resolution, lack of real-time schlieren image observation, non-parallel illumination, vibration sensitivity, and focusing difficulties. For most plasma investigations, it is no replacement for the laboratory instruments described earlier. More information on BOS can be found in [14, 36].

Figure 1g shows a "lens-and-grid" or "focusing" schlieren system (Ref. [16] Ch. 4) in which the light source is a large backlit source grid SG combined with a Fresnel lens FL. This grid is imaged by schlieren lens SL in plane CG, where a matching negative cutoff grid serves the purpose of a knife-edge. A relay lens RL then conveys the schlieren beam to camera $C$. Due to its large light source and aperture, this schlieren system has a narrow depth of focus at $S$, which is useful in isolating $\mathrm{S}$ from other refractive disturbances elsewhere along the optical path.

Finally, direct shadowgraphy [16], Fig. 1h, has also been used to observe plasma flows. In this extremely simple setup, light source LS and lens L project a parallel light beam through the schlieren object $\mathrm{S}$ and into the camera $\mathrm{C}$. The camera is not focused on $S$, however, but rather on plane $M$ at a distance $g$ from $S$. Refractions in $S$ naturally cast shadows in the resulting image, whose intensity variations indicate the Laplacian of the refractive index $n\left(\partial^{2} n / \partial x^{2}+\partial^{2} n / \partial y^{2}\right)$. To avoid blur, the shadowgraph light source must be quite small, typically less than $1 \mathrm{~mm}$ in size. 
The direct shadowgraph setup of Fig. 1h suffers from a small field-of-view that is limited by the camera lens diameter. A better approach is to adapt the z-type or lens-type instruments, Fig. 1a-b, for shadowgraphy by removing the knife-edge and focusing the camera lens on plane $M$ rather than S. This so-called "focused shadowgraph" approach is diagramed in Fig. 1i.

\section{Light sources}

Schlieren and shadowgraph light sources have traditionally included incandescentfilament bulbs, compact arc lamps, spark gaps, and flashlamps [16]. Recently LEDs have been added to this list [14], entirely replacing the dangerous spark gaps and providing broadband or monochromatic illumination inexpensively. Best of all, LEDs can be pulsed repeatedly at sub-microsecond intervals, which none of the other available light sources can do. Compact arc lamps, however, still outshine LEDs for high-speed imaging where continuous illumination is required.

Some plasmas produce intense self-illumination that can cause problems by overriding and washing out a schlieren image. A narrow opaque slit mask in the knife-edge plane can eliminate much of this stray light that is off-axis. Otherwise, the combination of a monochromatic light source and a corresponding bandpass filter BF ahead of the camera is often used in plasma studies to limit the effect of self-luminosity. Some LEDs have the advantage for this purpose of being monochromatic but not spatially coherent, as are lasers. When a laser is the schlieren light source, the usual razor-blade knife-edge cutoff cannot be used, and must be replace by a graded filter (Ref. [16] Ch. 5).

\section{Cameras}

Essentially all film cameras for schlieren and shadowgraphy have now been retired [14]. Figure 1 uses a Digital Single-Lens Reflex (DSLR) camera as an icon, and such consumer-grade cameras do serve well for both still and video schlieren imaging so long as microsecond exposures or high frame rates are not required. Most other consumer-grade cameras and camcorders are unsuitable due to fixed lenses with tiny apertures that would vignette the schlieren beam.

Beyond that, there is a broad range of scientific-grade CMOS and CCD cameras that can serve as schlieren cameras. Some of them can reach modestly-high frames rates of several hundred frames/s.

A new generation of digital high-speed cameras has replaced the film-based drum and rotating-mirror cameras of the twentieth century. These new cameras typically have CMOS sensors, megapixel resolution up to 20,000 frames/s, and frame rates as high as 5 million frames/s. Commercial examples of such cameras include the Photron Fastcam SA-Z and the Vision Research Phantom V2512. Their chief disadvantage is their cost, which is currently so high that a government or private grant is generally required to purchase one. It is worth mention that higher performance can be reached by some lab-made cameras, such as the one developed by the MIT Media Lab, which is able to reach a frame rate of around $10^{12}$ frame/s [37]. 


\section{Schlieren imaging for the study of atmospheric pressure plasmas}

Schlieren imaging of atmospheric pressure plasmas is a powerful technique to address fundamental studies concerning their fluid-dynamics as well as to study, characterize and optimize processes assisted by them. Moreover, being completely non-intrusive, schlieren imaging presents a major advantage over other techniques, such as Particle Image Velocimetry (PIV), that use tracers, which could be affected by the electromagnetic field of the plasma. Since atmospheric pressure plasma sources show marked differences among themselves, the schlieren setups should be tuned case-by-case in order to achieve a thorough characterization of the plasma fluid-dynamics occurring in the processes under study.

From this perspective, even if not comprehensive of all the existing atmospheric pressure plasmas, three main categories of sources and processes have been identified among research papers in which schlieren imaging is performed on atmospheric pressure plasmas and the schlieren setups therein adopted are discussed in this work: non-equilibrium Atmospheric Pressure Plasma Jets (APPJs), plasma actuators for flow control and thermal plasma sources. For each category, a selection of the published work has been made in order to present useful considerations, suggestions and practical examples on the use of schlieren imaging to obtain qualitative and quantitative information. These solutions may be suitably implemented in the readers' optical setups to achieve new and optimal results during their research activities.

\section{Non-equilibrium APPJs}

In order to satisfy the requirements and demands involved in different biomedical and industrial fields for devices able to promote the production of different reactive and charged species at ambient temperature and deliver them into the surrounding environment, jet configurations were taken into account, leading to the development of the cold Atmospheric Pressure Plasma Jets (APPJs). In APPJs, usually working with noble gases such as helium, neon or argon, a plasma plume flows from the nozzle into the surrounding atmosphere.

Although devices with jet-like flows have been well studied in the industrial field, a thorough description of their behaviour requires the use of complex physical models, necessarily supported by direct experimental measurements [38], where schlieren imaging can play an important role for the visualization and interpretation of fluid-dynamic phenomena. Moreover, since plasma is a reactive and conductive gas, the fluid-dynamics of an APPJ may be strongly affected by the chemical and electrodynamics forces, driven by different physical quantities such as reactive and charged species concentrations, gas temperatures and electric fields. Under this view, schlieren imaging has been adopted to obtain reliable measurements of the flow characteristics of APPJs with the aim to evaluate reactive species transport and process feasibility [39].

Considering the best practices in the experimental setup of the schlieren imaging of APPJ, it is worth highlighting that the knife-edge is generally aligned with the axis of the jet produced by the plasma source [28, 40-56], because the highest gradients of refractive index in the jet region are radial. Moreover, some groups have suggested 
investigating APPJs placed in a horizontal orientation in order to emphasize buoyancy effects due to the different density of the working gas (e.g. helium, argon...) in comparison with the surrounding air $[40,41]$.

Regarding the choice of the light source for schlieren analysis of APPJ, no relevant trends can be found. Thanks to the low luminous intensity of the plasma plume produced by APPJs, there is a wide availability of light sources ranging from monochromatic lasers to broad-band- spectra lamps. As a general indication, on one hand, light sources with a narrow spectral range, such as LED or laser [28, 41-47], lead to optimization of the light path because the interaction between radiation and optical components (lens, mirrors, detectors...) is a function of wavelength and leads to noise reduction from the self-luminosity of the plasma plume (for example using green light); on the other hand, high intensity lamps, such as xenon or mercury arc lamps [48-51,54], characterized by a continuum spectrum, guarantee that a great many photons reach the detector, enhancing the resolution of collected images and allowing the use of high-speed cameras for time-resolved investigation.

Schlieren imaging has easily allowed direct investigation of how the jet flow of APPJs can be affected by the ignition of plasma, inducing a displacement of the transition point from laminar to turbulent flow $[49,52]$.

Furthermore, the understanding of plasma plume fluid-dynamics can be further enhanced if schlieren imaging is coupled with other imaging diagnostic techniques. For example, since the plasma plume itself emits luminous radiation, the interplay between the characteristics of the luminous plasma plume and fluid-dynamic phenomena can be investigated through a combination of standard or intensified camera (ICCD) imaging and schlieren visualization. In particular, the use of an ICCD camera is recommended when it is necessary to study short-time-scale events, such as micro- and nano-pulsed discharges, or a plasma plume with a low selfluminosity. Although several configurations of APPJ exist that can be driven by different voltage and frequency waveforms, conventional and schlieren imaging can be easily used for different purposes, such as to find the operating conditions that maximize the elongation of the plume [41, 53], to evaluate modification of the plasma plume induced by the presence of a nearby conductive substrate [43, 57], or to study the interaction of multiple plasma plumes in an APPJ-array [42]. Moreover, using ICCD and schlieren images, Boselli et al. [48, 54] highlighted how the branching of the plasma plume structure of a ns-pulsed APPJ occurs at transition to turbulence and the rare-gas concentration drops due to mixing with the ambient air, as represented in Fig. 2.

With the purpose of studying the temporal evolution of turbulence generated by pulsed APPJ, different groups have improved schlieren imaging by adopting high-speed cameras $[45,48,50,54,56]$. In particular, studying APPJs driven by pulse generators at low frequency (lower than $1 \mathrm{kHz}$ ), some research groups [45, 48] have observed how the ignition of plasma discharge can develop a turbulent front propagating downstream through the jet.

Absorption and emission spectroscopy are two diagnostic techniques that can yield significant results when coupled with schlieren imaging. Indeed, through this coupling the researchers can evaluate the spatial distribution and temporal evolution of emitting species and the temperature field of APPJs [54]. 


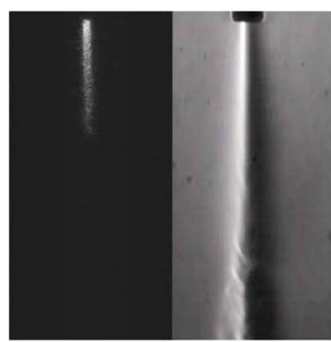

$\mathrm{PV}=17 \mathrm{kV}-\mathrm{PRF}=1000 \mathrm{H} /$

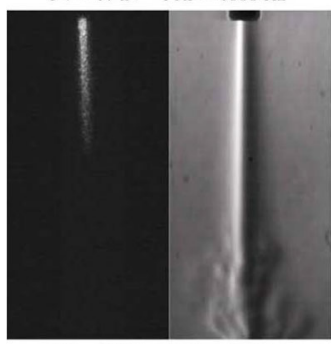

$\mathrm{PV}=17 \mathrm{kV}-\mathrm{PRF}=125 \mathrm{~Hz}$

Fig. 2 Comparison of synchronized ICCD (left) and schlieren (right) images of a helium APPJ for different values of peak voltage (PV) and pulse repetition frequency (PRF). Flow rate set at 3 SLPM of He for all cases [54] C 2015 IEEE. Reprinted, with permission, from IEEE Transactions on Plasma Science

Because of the variety of APPJ source configurations, the development of a numerical model that can well describe and simulate the fluid-dynamics and spatial distribution of chemical reactive species of an APPJ is considered a crucial step to approach the needs of the industrial and biomedical fields. Schlieren imaging with other diagnostic techniques, such as thermocouple measurements and infrared thermal imaging, can be used to validate and optimize the computational models for the simulation of APPJ flows by comparing numerical and experimental data [40, 47]. With a similar approach, Papadopoulos et al. [44], through the merging of a numerical model and schlieren results (an example is given in Fig. 3), investigated the interaction between fluid-dynamic and electro-dynamic forces in the effluent of an APPJ, showing that, in their experimental setup, the flow field alteration is mainly affected by the electro-dynamics, rather than by local gas temperature variations.

Moreover, as reported in [26], schlieren imaging can be also used to obtain quantitative information: Schmidt-Bleker et al. [46] have proposed a method to find the distribution of species density, gas temperature and calorimetric power of APPJs, combining schlieren images with computational fluid-dynamics simulations. These authors have suggested using a dielectric filter with a transmission wavelength of $550 \mathrm{~nm}$ and a full width at half maximum of $10 \mathrm{~nm}$ in front of the camera in order to eliminate the self-luminosity of the plasma and the background radiation. It is worth mentioning the paper appendix, where a few aspects of quantitative schlieren are presented, such as sensitivity determination and some advantages and disadvantages of using a circular aperture.

Following this approach, recently Chamorro et al. [28], derived the temporallyaveraged values of the gas temperature of the jet by processing the gray-level contrast values of digital schlieren images and coupling them to a simplified kinetic model of the jet effluent region. With their setup, the authors managed to obtain radial profiles of contrast in a "plasma off" condition that were quite symmetric, while asymmetries 


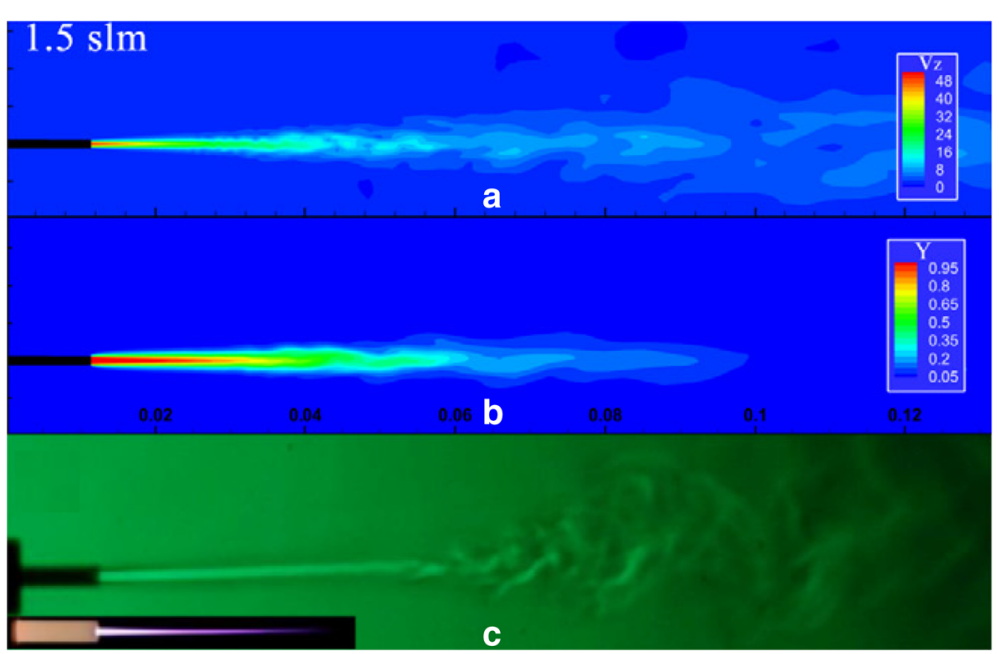

Fig. 3 Numerical simulations of (a) the axial velocity $V_{z}\left(m^{-1}\right)$ and (b) helium mass fraction $Y$, compared with (c) an experimental schlieren image, for $1.5 \mathrm{slpm}$ of He flow rate and the plasma in operation $\left(20 \mathrm{kV}_{\mathrm{p}-\mathrm{p}}\right)$. Schlieren imaging was performed in a Z-type configuration setup with a high-power green light-emitting diode, two spherical mirrors and a Foucault knife-edge tester [44] ๑ Deutsche Physikalische Gesellschaft. Reproduced by permission of IOP Publishing. CC BY-NC-SA

occurred in "plasma on" conditions, probably induced by an asymmetric discharge generation. These departures from radial symmetry of contrast can perturb the data elaboration when Abel inversion is performed, especially towards the jet axis. Nonetheless, the comparison of the calculated temperature values with k-type thermocouple temperature measurements shows an error below $5 \mathrm{~K}$.

By means of schlieren imaging, Bradley and et al. [51] have investigated the relationship between the region of impact of the APPJ effluent transporting the reactive species and the surface affected by a wettability modification. In particular, they have pointed out how the area with an increased water wettability, quantifiable through the measurement of the water contact angle (WCA), is larger than the area reached by the visible plasma plume. The same authors have also studied also the increase of hydrophilicity induced by a APPJ with the plasma plume and gas outflow directed parallel to and just above the sample surface; they have shown how a detectable reduction of the WCA occurs over all the surface covered by the turbulent part of the APPJ effluent, although no impinging point between the visible plasma plume and the targeted surface was present in this particular setup [50].

Moreover, considering that APPJs have also found great interest in the last decade in the field of liquid treatment, it is worth highlighting how Shimizu et al. [55] applied schlieren imaging for the characterization of thermal flow fields and transport of chemically reactive species in gas and liquid phases, pursuing a more thorough understanding of the transport mechanisms occurring across the gas-liquid interface. Schlieren images, coupled with PIV imaging (for measurement of flow patterns in the liquid phase), showed how APPJ effluent induces a circulating flow in the liquid and the chemical components generated in the gas phase are dissolved and carried by convection in the liquid (Fig. 4).

Even if not strictly correlated to APPJs, the use of schlieren imaging performed with an ICCD camera and a laser diode can be suitable for studies with extremely high 


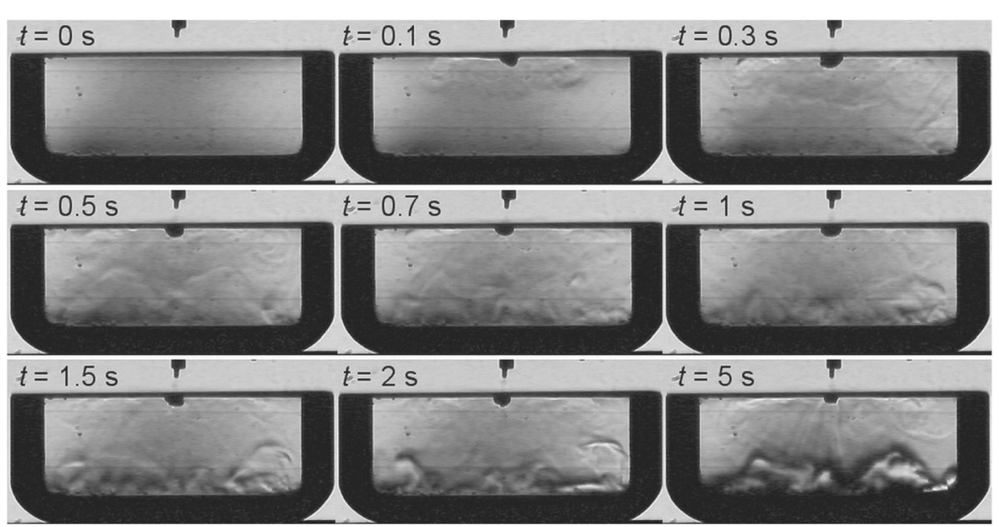

Fig. 4 Thermal field in water due to APPJ impingement, measured by schlieren imaging. At $t=0 \mathrm{~s}$ the plasma discharge was initiated. The APPJ nozzle is shown at top center of each image [55] () Deutsche Physikalische Gesellschaft. Reproduced by permission of IOP Publishing. CC BY-NC-SA

temporal resolution of plasmas in liquid phase. This setup, for example, allowed investigators to detect the formation of a negative pressure region in the vicinity of a highvoltage pin electrode driven by nanosecond pulses and the following formation of a shock wave [58]. The phenomenon, attributed to the electrostriction mechanism, may play a key role in the development of breakdown in the liquid phase. An et al. used a similar setup with a different purpose: to study the fast development and propagation of primary and secondary streamers and analyse their morphology (e.g. bush-like and filamentary) in transparent liquids [59].

\section{Plasma actuators for flow control}

Being a characterization technique of common use in aerodynamics, schlieren imaging has been widely employed to study the fluid-dynamic phenomena induced by plasma actuators, which are designed to affect the surrounding atmosphere through different mechanisms, like the production of ionic wind, the alteration of the laminar-turbulent transition location in a boundary layer, the suppression of flow separation, and the formation of vortices and pressure waves. Different types of plasma actuators have been adopted to achieve desired fluid-dynamic effects, such as surface Dielectric Barrier Discharges (DBDs), Sliding Discharges (SDs) and sparkjets [6].

Most of the studies on plasma actuators present conventional schlieren optics, such as z-type systems with or without flat folding mirrors [60-76], as are used in wind tunnel facilities. The knife edge is employed both in the horizontal and vertical positions and all the different light sources previously discussed have been adopted also for the characterization of plasma actuators, like lasers [35, 66, 77], LED lamps of a few $\mathrm{W}$ power [67, 69, 78-81], pulsed lights [60, 64, 70-72, 82] and arc or halogen lamps [61-63, 65, 74-76, 83, 84].

As reported in section 1.2, only Biganzoli et al. suggested using BOS to characterize plasma actuators [35]. They show some preliminary results on an AC-surface DBD and the ionic wind induced by the source. BOS permits simultaneous observation of the gradient of refractive index in two orthogonal directions, allowing one to portray fluiddynamic phenomena characterized by a $2 \mathrm{D}$ field of $n$, while standard schlieren setups 
are limited to the component perpendicular to the knife edge when the usual knifeedge is used as a cutoff. However, as described in section 1.2, BOS suffers from several drawbacks that can negatively affect the quality of the results, and therefore its use on plasma actuators remains marginal.

Most of the studies on plasma actuators have used schlieren imaging mainly to qualitatively investigate the induced fluid-dynamic phenomena $[35,61-63,65,66$, 68, 73-77, 84-87]. Some research groups have used phase-locked schlieren imaging to perform their studies, since in this technique the acquisition is synchronized to a specific instant with regard to the frequency of the discharge, thus allowing one to produce more accurate images obtained by the average of several acquisitions. This can also be useful to obtain important data, such as the speed of induced pressure waves and jet flows [62-64, 70, 72, 78-80, 82, 85]. The drawback of this method is that the stochastic flow perturbations induced by the discharge are neglected, being treated as noise, as reported by Leonov et al. [77].

To further highlight the effects of the plasma discharge on the gas density, Komuro et al. [81] have post-processed the acquired frames by subtracting the background signal taken before plasma ignition. Furthermore, they realized some "streak pictures" from the schlieren frames in order to easily evaluate the spatio-temporal evolution of the studied phenomenon along three spatial axes defined with respect to the profile of the airfoil employed in their experiments.

An original setup to enhance the quality of the schlieren images in a wind tunnel facility has been presented by Hai et al., focused on the study of the flow separation control on an airfoil model through nanosecond-pulsed DBDs [69]. They heated the wall of the airfoil model, placed inside the wind tunnel, in order to create a difference of temperature between the airfoil model and the main flow, that resulted in an increased difference of the density between the air impinging on the airfoil and the undisturbed flow. Using this setup, they revealed that pressure waves were not relevant to the delay of flow separation, which was regulated by viscous disturbances, i.e. the heated air with different viscosity.

Some research groups have used schlieren images to compare simulated and experimental results and to validate numerical models, from which quantitative information on the investigated phenomena can be obtained, especially on the aerodynamic forces [61, 68, 71, 83, 88].

Other studies obtained quantitative data directly from the schlieren frames. Sosa et al. studied the flow induced by a SD or a surface DBD, using two vertical helium jets placed near to the source (Fig. 5, upper) in order to gain contrast in the obtained images [73, 74, 89]. Here the helium serves as a tracer, having a lower density than the surrounding air and also enabling observation of the effect of the induced ionic wind outside the central region of the SD without significantly affecting the plasma discharge characteristics. Moreover, the authors developed and applied the Schlieren Image Velocimetry technique, which is a dense motion estimator to extract velocity fields from the obtained frames (Fig. 5, lower) [73, 74]. This technique is based on the minimization of an energy function consisting of a data term, which relies on the physical relation between the luminance and the fluid density gradient, and a regularization term. More details on the principles of this technique can be found in [90]. 

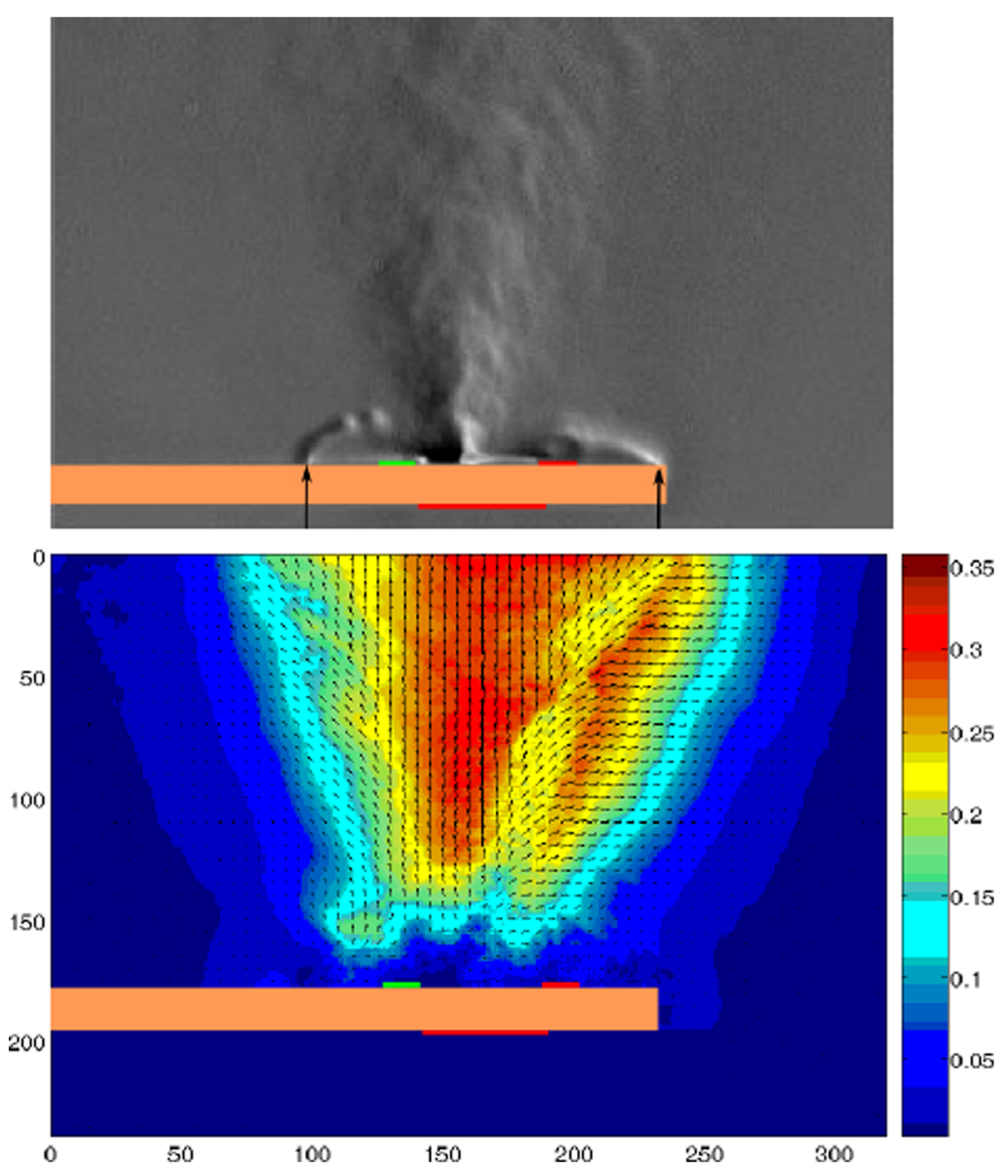

Fig. 5 (upper) Schlieren image of the SD, with the black arrows indicating the vertical He jets injection points; (lower) time averaged velocity field obtained by Schlieren Image Velocimetry in $\mathrm{m} / \mathrm{s}$ [74] $\odot$ 2009 IEEE. Reprinted, with permission, from IEEE Transactions on Dielectrics and Electrical Insulation

Another method to obtain the velocity fields from schlieren frames was proposed by Cristofolini et al., based on an intensity integral function and its relationship with the gas density [61]. The velocity profiles were calculated from the pixel intensities of the schlieren images and then compared with the profiles obtained from pitot tube measurements, showing a good agreement for most of the conditions studied, as also seen in the work of Gao et al. using the same method [67].

Correale et al. used an image-processing algorithm, based on a threshold set on the light intensity, to estimate the heated region produced by the discharge of a nanosecond-pulsed DBDs (as shown in Fig. 6), thus evaluating the distribution of the energy released by the nanosecond-pulses [64].

Takashima et al. have studied the pressure waves produced by a nanosecondpulse DBDs on planar or cylindrical surfaces using a technique different from the approach of post-processing the obtained schlieren frames [78-80]. In this case, the density gradient of the waves was inferred from the schlieren images using a pair of wedge-shaped glass prisms to perform a calibration of the schlieren system, as these windows can be rotated in order to change the schlieren signal intensity. The use of optical prisms or wedges of very small angle as schlieren calibrators dates back to 1957 [91]. 


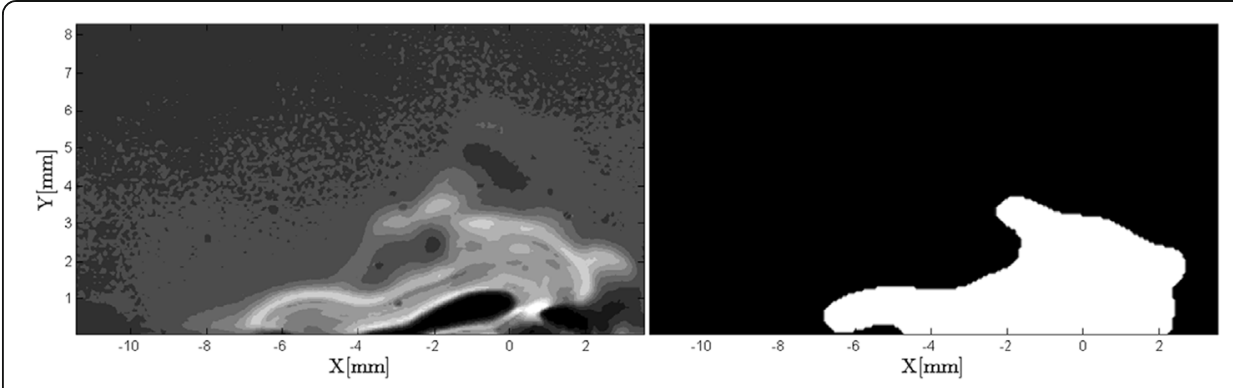

Fig. 6 Unprocessed schlieren frame of the discharge of a nanosecond-pulsed DBD (left) and the same frame in which the heated region produced by the discharge is estimated by means of an algorithm processing the light intensity (right) [64] (c) Deutsche Physikalische Gesellschaft. Reproduced by permission of IOP Publishing. CC BY-NC-SA

\section{Thermal plasmas}

Compared to the more recent non-equilibrium plasma sources presented in the previous sections, which date back as far as twenty years, thermal plasmas have quite a longer history of industrial applications, such as material synthesis [8], thermal spray [9], plasma cutting [92] and welding [93]. Accordingly, several examples of schlieren imaging investigations of these plasma sources are reported since the early 50's [94], even without the use of modern light sources and digital cameras.

Starting from the end of the 80's, a few investigations on thermal plasma spray by shadowgraphy and/or schlieren imaging are reported, in order to evaluate air entrainment in the plasma jet due to the transition from laminar to turbulent behavior, as the presence of air can oxidize the particles injected in the plasma, affecting the quality of the resulting coating. These papers present mainly results obtained by other diagnostic tools, such as laser doppler anemometry, coherent anti-Stokes Raman spectroscopy [95], and enthalpy probe and mass spectrometry measurements [96, 97]. Shadowgraphy and schlieren imaging are used to support the visualization of the plasma torch outflow for different operating conditions. Moreover, almost no description of the adopted imaging setup is provided. Pfender et al. [95] used a shadowgraphy system with an image of $75 \mathrm{~mm}$ diameter to visualize a $350 \mathrm{~A}$ argon plasma jet in air. By increasing the gas flow rate from 305.8 to 1834.9 slpm the onset of the transition region from laminar to turbulent flow moves upstream, with a reduction in the size of the turbulent eddies. The reported images are affected by an intense plasma jet light emission which partially covers the jet outflow, probably due to the fact that no filters have been used and the exposure time of the camera was not short enough to dim the light emitted from the plasma. Russ et al. [97] managed to overcome the plasma intensity of a $450 \mathrm{~A}$ argon jet by using a laser shadowgraph system with a pulsed argon ion laser ( $1 \mathrm{~W}$ at $514.5 \mathrm{~nm}$, with approximately $0.1 \mathrm{~ms}$ pulse duration) and an exposure of $1 \mu \mathrm{s}$, allowing them to investigate the region close to the nozzle exit. In the same paper, the behavior of a nozzle that reduced the outlet turbulence through a flow straightener was investigated by schlieren imaging, with heated air and helium (without plasma), for different flow rates, temperatures and turbulence conditions. The observed behavior of the jet was not confirmed by shadowgraphy of an operating plasma torch with similar flow conditions (in terms of relative difference of density between the jet and the surrounding air). The authors suggested that the erratic behavior of the plasma spray arc, which is commonly 
subjected to swirling gas flow and to anodic arc reattachment, was a possible explanation for the difference.

Raghu et al. [98] used a focusing schlieren setup (Fig. 1g) and a strobe light source to compare the behavior of two different plasma spray guns, respectively gas- and waterstabilized, operated at approximately $400 \mathrm{~A}$. The gas-stabilized torch used its nozzle walls as anode, while the water-stabilized torch had an external rotating anode at the exit of its nozzle. While the focusing schlieren setup should have a narrow depth of field and the ability to minimize the plasma glow, the reported images are quite blurry and with an evident overexposure due to the plasma emission. It was possible to measure a half-angle of the jet spread of $20^{\circ}$ and to verify that the water-stabilized plasma gun had a more asymmetric behavior than the gas-stabilized gun due to the external rotating anode.

An $\Omega$-type setup in a controlled pressure plasma reactor has been used by Henne et al. [99] for the characterization of three modified-anode nozzles designed for a commercial plasma gun, also supported by enthalpy probe, mass spectrometry and in-flight particle measurements. They used a Xe-pumped Nd-YAG pulsed laser $(532 \mathrm{~nm}$, with pulse duration of 5-7 ns) as light source and a small circular aperture in place of a knife-edge cutoff. With just $100 \mathrm{kPa}$ of pressure inside the reaction chamber the refractive index gradient of the jet boundary was at the limit of detectability of the schlieren imaging setup. By gradually increasing the pressure up to atmospheric the gradients became more evident. At $30 \mathrm{~kW}$, with $45 \mathrm{slpm}$ of argon and at low pressure, the jet is laminar, but changes to turbulent flow with pressures higher than $20 \mathrm{kPa}$.

A recent paper by Horba et al. [100] investigated a 500 A water-stabilized plasma torch by means of a lens-type schlieren imaging setup, using a pulsed Nd laser (540 nm, $35 \mathrm{~ns}$ of pulse width) and probably a circular aperture schlieren cutoff (not specified in the paper). In this paper the presence of "plasma structures" (small bodies of plasma within the surrounding cold atmosphere) ejected from the plasma jet boundary is also verified by electrical probe measurements.

Concerning atmospheric pressure radio frequency induction plasmas, it seems that there are no studies involving schlieren imaging with plate powers higher than a few $\mathrm{kW}$. The use of such plasmas is usually associated with reaction chambers, which would require several complex modifications in order to allow the schlieren light beam to reach the region of interest; moreover, the interaction of the plasma jet with cold surrounding air is prevented by the reaction chamber walls, with the reactor usually filled with the same gas that operates the plasma torch. However, torches for Inductively Coupled Plasma-Optical Emission Spectroscopy (ICP-OES) in the $0.3-2 \mathrm{~kW}$ power range have been recently investigated by a couple of research groups. Nagulin et al. [101] presented results on high-speed schlieren imaging of the plasma ignition and operation of a standard $2 \mathrm{~kW}$ ICP torch for OES and compared with 3D modelling results. A lens-type schlieren imaging setup was used, with a $450 \mathrm{~W}$ arc Xe lamp and with both horizontal and vertical knife edge orientations. The authors compared modelling results of temperature distribution directly to schlieren frames, arguably concluding that they were "almost identical". However, the temperature distribution cannot be directly used for comparison with schlieren frames, as refractive index, (proportional to density) and temperature have an inversely proportional correlation. Moreover, temperature profiles lack information on pressure and composition distribution, which also affect refractive index. The inaccuracy of this approach is confirmed by the several 
evident differences between the schlieren frames and the temperature profiles shown in the paper. Also the high-speed schlieren frames obtained with the knife edge oriented perpendicularly to the ICP axis are quite blurred, even compared to other images in the same paper.

A similar ICP torch was investigated by Boselli et al. [102] with a z-type schlieren setup, and also using direct high-speed imaging, correlating schlieren and high-speed imaging for different feed gas flow rates and plate power levels. The use of schlieren imaging also allowed them to analyze the outflow of a quenching device, designed for the production of reactive species for biomedical applications [103] and placed at the ICP torch outlet. The use of a knife edge perpendicular to the ICP axis allowed the imaging of the device outflow as it impinged on an agar plate, even though it was partially covered by a curtain of heated argon rising due to buoyancy. Traldi et al. [104] analyzed by schlieren imaging an ICP jet either impinging on a cylindrical substrate or in the free flow regime. Schlieren imaging allowed to evaluate the strong influence of the feed gas flow rates on the shape of the plasma plume and how the presence of the substrate affects the fluid-dynamics of the effluents. The frames obtained were correlated with simulated velocity vectors coming from $3 \mathrm{D}$ modeling results, showing that the fluid-dynamic characteristics of the plasma plume were properly reproduced by simulation.

In plasma cutting technology, cut quality can be strongly affected by a turbulent interaction between the effluent jet (the plasma arc column surrounded by its cold gas shroud), and the surrounding atmosphere downstream the torch nozzle; highspeed and schlieren imaging have been adopted to investigate this behavior by several groups [92]. Bemis and Settles [105] performed the first study on oxygen plasma cutting of mild steel by schlieren imaging. A 30 A commercial plasma torch was investigated with a lens-type schlieren setup, a vertical knife edge and a microsecond-flash lamp synchronized with a video camera. In order to improve the image contrast, several different filters were used. The turbulence generated by the arc-air interaction is shown over and below the steel plate, and even well-focused liquid metal ejections and trails are visible.

Kim et al. [106] used a z-type setup for comparing the performance of two different 60 A plasma torch consumable sets. A $700 \mathrm{~W}$ mercury-arc light source was used, with a first neutral filter to control the background light, a second filter to control the arc column intensity and, finally, a circular cutoff instead of a knifeedge. Schlieren imaging was used by these authors mostly to compare in terms of width and symmetry of the arc its stability for two consumables sets, using line intensity analysis, a fast Fourier transform of the video signal and a log-Gabor filter to evaluate symmetry [107]. The same investigative approach has been used by the same authors to analyze the increase in constriction and symmetry of the arc due to the use of micro-jets [107].

Cantoro et al. [108] also used windowed standard deviation and the Generalized Autoregressive Conditional Heteroskedasticity (GARCH) approach to map the variance intensity of the turbulence in each region close to the plasma jet. They used a $450 \mathrm{~W}$ xenon arc lamp and a z-type schlieren setup to analyze a 25 A cutting torch. Prevosto et al. [27] proposed a technique based on the reconstruction by Abel inversion of refractive index, arc temperature and ionized-species density profiles. Their 
reconstruction method requires knowledge of the pressure in the region of investigation; so they focused at $3.5 \mathrm{~mm}$ below the nozzle on the torch axis, far beyond the shock front of a typical plasma cutting torch, and a position where atmospheric pressure could be assumed. They investigated a 30 A cutting torch on a rotating anode by using a z-type schlieren system with a vertical knife edge and a $20 \mathrm{~mW}$ continuous laser source (main wavelength $632.8 \mathrm{~nm}$ ) with a bandwidth filter to block the arc light. The same authors [109] improved their technique by using an additional laser source (main wavelength $514 \mathrm{~nm}$ ), another bandwidth filter and a beam-splitter to allow the use of both laser sources. As the refractive index of the electrons is strongly dependent on the light wavelength of the probe beam, using two different light sources and modified two-temperature Saha equations the authors were able to separately evaluate the electron and the heavy particle temperatures.

Schlieren imaging has been used as valuable research tool in plasma welding in order to visualize the flow of the shield gas which surrounds the welding arc, protecting the weld pool from air intrusions [93]. To our knowledge, the first paper on schlieren photography of Gas Tungsten Arc Welding (GTAW) was published by Moen and Gibson in 1952 [94]. The authors used a $1.25 \mu$ s shutter and a $5 \mu \mathrm{s}$ flash tube to investigate the behavior of argon and helium as shield gases, with a z-type schlieren setup and several different welding positions. In each picture the arc region is affected by light overexposure, but nonetheless by using a knife-edge in the horizontal position it was possible to verify the coverage of the shielding gas on the welded metal plate. Another paper published in the late 70's involving the schlieren imaging of GTAW and Gas Metal Arc Welding (GMAW) sources was written by Kiyohara et al. [110]. The authors solved the arc light overexposure issue by using a He-Ne laser source (main wavelength $632.8 \mathrm{~nm}$ ) with a bandpass filter and an $\Omega$-type optical setup, allowing them to analyze the interface between the shield gas and surrounding air for different operating currents and shield gas flow rates. Unfortunately, this paper is only available in Japanese so several details on the setup and the results are not easily understandable.

More recently Schnick et al. [111] used a z-type schlieren system to investigate a 100 A GTAW source. They have shown that, by increasing the shield gas flow rate, there is a change from laminar to turbulent behavior which increases the air mixing in the weld puddle and negatively affects the welding bead quality.

An extended study of the potential of schlieren imaging has been performed by Siewert et al. [93]. The effect of several different solutions, such as different knife-edge orientations and choices of light source, are presented for GTAW, GMAW and plasma-arc welding. A recent work by Bitharas et al. [112] investigated the effect of varying the composition of the shield gas by an alternating Ar-He supply during a 100 A GTAW process. They adopted a z-type schlieren setup and a 10,000 lm LED source with a $633 \mathrm{~nm}$ bandpass filter and a vertical knife edge.

\section{Conclusions}

In this paper, the potential of schlieren imaging for atmospheric-pressure plasma diagnostics has been presented. Thanks to schlieren imaging, much information can be obtained on the fluid-dynamic phenomena occurring in plasma processes in a non-intrusive way. The mixing of plasma-source effluent with the surrounding 
air and the generation of viscous disturbances which delay flow separation in a plasma actuator are just two examples of the phenomena observable by this technique. In the first part of the paper, schlieren theory and instruments were presented together with the main characteristics of most common schlieren setups and their drawbacks, which have to be taken into account in the choice of which schlieren setup to use. In the second part of the paper several practical examples exploiting the advantages of schlieren imaging and suggestions on how to address the experimental issues typically encountered when using schlieren imaging as a plasma diagnostic were reported, focusing on APPJs, plasma actuators for flow control and thermal plasma sources. This work reveals that schlieren imaging is a powerful diagnostic tool, not only for fundamental research but also for processes optimization, and that it is important for numerous plasma applications.

\begin{abstract}
Abbreviations
APPJs: Atmospheric Pressure Plasma Jets; BOS: Background-Oriented Schlieren; DBD: Dielectric Barrier Discharge; GARCH: Generalized Autoregressive Conditional Heteroskedasticity; GMAW: Gas Metal Arc Welding; GTAW: Gas Tungsten Arc Welding; ICCD: Intensified Charge-Coupled Device; ICP-OES: Inductively Coupled Plasma-Optical Emission Spectroscopy; PIV: Particle Image Velocimetry; PRF: Pulse repetition frequency; PV: Peak voltage; SD: Sliding Discharge; WCA: Water contact angle
\end{abstract}

Availability of data and materials

Data sharing not applicable to this article as no datasets were generated or analysed during the current study.

Authors' contributions

All authors took part in discussions on the content of the manuscript. All authors read and approved the final manuscript.

Competing interests

The authors declare that they have no competing interests.

\title{
Publisher's Note
}

Springer Nature remains neutral with regard to jurisdictional claims in published maps and institutional affiliations.

\section{Author details}

${ }^{1}$ Department of Industrial Engineering, Alma Mater Studiorum - Università di Bologna, Viale del Risorgimento 2, 40136 Bologna, Italy. ${ }^{2}$ Center for Industrial Research - Advanced Applications in Mechanical Engineering and Materials Technology, Alma Mater Studiorum - Università di Bologna, Viale del Risorgimento 2, 40136 Bologna, Italy. ${ }^{3}$ Department for Life Quality Studies, Alma Mater Studiorum - Università di Bologna, Corso d'Augusto 237, 47921 Rimini, Italy. ${ }^{4}$ FloViz Inc., Port Matilda, PA 16870, USA.

Received: 31 January 2018 Accepted: 24 April 2018

Published online: 10 May 2018

\section{References}

1. Samukawa S, Hori M, Rauf S, Tachibana K, Bruggeman P, Kroesen G et al (2012) The 2012 plasma roadmap. J Phys D Appl Phys 45(25):253001

2. Adamovich IV, Baalrud SD, Bogaerts A, Bruggeman PJ, Cappelli M, Colombo V et al (2017) The 2017 plasma roadmap: low temperature plasma science and technology. J Phys D Appl Phys 50(32):323001

3. Fridman A (2008) Plasma chemistry. Cambrige University Press, Cambridge

4. Parvulescu VI, Magureanu M, Lukes P (2012) Plasma chemistry and catalysis in gases and liquids. Plasma chemistry and catalysis in gases and liquids. WILEY-VCH Verlag GmbH \& Co. KGaA, Weinheim

5. Laroussi M, Kong MG, Morfill GE, Stolz W (2012) Plasma medicine: applications of low-temperature gas plasmas in medicine and biology. Cambridge University Press, Cambridge

6. Moreau E (2007) Airflow control by non-thermal plasma actuators. J Phys D Appl Phys 40:605-636

7. Wang J-J, Choi K-S, Feng L-H, Jukes TN, Whalley RD (2013) Recent developments in DBD plasma flow control. Prog Aerosp Sci Elsevier 62:52-78

8. Boulos MI, Fauchais P, Pfender E (2013) Thermal plasmas: fundamentals and applications. Springer Science \& Business Media, New York

9. Fauchais PL, Heberlein JVR, Boulos MI (2014) Thermal spray fundamentals: from powder to part. Springer Science +Business Media, New York

10. Zhukov MF, Zasypkin IM (2007) Thermal plasma torches design, characteristics, applications. Cambridge International Science Publishing Ltd, Cambridge

11. Weltmann K-D, Kindel E, Brandenburg R, Meyer C, Bussiahn R, Wilke C et al (2009) Atmospheric pressure plasma jet for medical therapy: plasma parameters and risk estimation. Contrib Plasma Physics 49(9):631-640 
12. Deng $X L$, Nikiforov $A Y$, Vanraes $P$, Leys C (2013) Direct current plasma jet at atmospheric pressure operating in nitrogen and air. J Appl Phys 113(2):23305

13. Van Gessel AFH, Hrycak B, Jasiński M, Mizeraczyk J, Van Der Mullen JJAM, Bruggeman PJ (2013) Temperature and NO density measurements by LIF and OES on an atmospheric pressure plasma jet. J Phys D Appl Phys 46(9):95201

14. Settles GS, Hargather MJ (2017) A review of recent developments in Schlieren and shadowgraph techniques. Meas Sci Technol 28(4):42001

15. Gherardi M, Puač N, Marić D, Stancampiano A, Malović G, Colombo V et al (2015) Practical and theoretical considerations on the use of ICCD imaging for the characterization of non-equilibrium plasmas. Plasma Sources Sci Technol 24(6):64004

16. Settles GS (2001) Schlieren and Shadowgraph Techniques: Visualizing Phenomena in Transparent Media. SpringerVerlag. Springer Science \& Business Media, New York

17. Krehl P, Engemann S (1995) August Toepler - the first who visualized shock waves. Shock Waves 5(1-2):1-18

18. Schardin H (1942) Die Schlierenverfahren und ihre anwendungen. Ergebnisse der exakten. Naturwissenschaften 20:303-439

19. Settles GS (1985) Colour-coding Schlieren techniques for the optical study of heat and fluid flow. Int J Heat Fluid Flow 6(1):3-15

20. Merzkirch W (1987) Flow visualization, 2nd edn. Academic Press, New York

21. Meyer-Arendt JR (1992) Selected papers on Schlieren optics, vol MS61. SPIE, Bellingham

22. Holder DW, North RJ (1963) Schlieren methods, NPL notes on applied science no. 31. Her Majesty's Stationery Office, London

23. Weinberg FJ (1963) Optics of flames: including methods for the study of refractive index fields in combustion and aerodynamics. Butterworths, London

24. Vasiliev LA (1971) In: Baruch A (ed) Schlieren methods. Israel Program for Scientific Translations, New York

25. Tropea C, Yarin AL, Foss JF (2007) Springer handbook of experimental fluid mechanics. Springer-Verlag, Berlin

26. Hargather MJ, Settles GS (2012) A comparison of three quantitative Schlieren techniques. Opt Lasers Eng Elsevier 50(1):8-17

27. Prevosto L, Artana G, Mancinelli B, Kelly H (2010) Schlieren technique applied to the arc temperature measurement in a high energy density cutting torch. J Appl Phys 107(2):23304

28. Chamorro JC, Prevosto L, Cejas E, Fischfeld G, Kelly H, Mancinelli B (2018) Ambient species density and gas temperature radial profiles derived from a Schlieren technique in a low-frequency non-thermal oxygen plasma jet. Plasma Chem Plasma Process 38(1):45-61

29. Kelly DC (1963) Plasma equation of state. Am J Phys 31(11):827-828

30. Colombo V, Ghedini E, Sanibondi P (2008) Thermodynamic and transport properties in non-equilibrium argon, oxygen and nitrogen thermal plasmas. Prog Nucl Energy 50(8):921-933

31. Bracewell R (2003) Fourier analysis and imaging. Kluwer Academic, New York

32. Agrawal AK, Albers BW, Griffin DW (1999) Abel inversion of deflectometric measurements in dynamic flows. Appl Opt 38(15):3394-3398

33. Ishino Y, Hayashi N, Bt Abd Razak IF, Kato T, Kurimoto Y, Saiki Y (2016) 3D-CT(computer tomography) measurement of an instantaneous density distribution of turbulent flames with a multi-directional quantitative Schlieren camera (reconstructions of high-speed premixed burner flames with different flow velocities). Flow, Turbul Combust 96(3):819-835

34. Dall HE (1947) A null test for paraboloids. J Br Astron Assoc 57(5):201-205

35. Biganzoli I, Capone C, Barni R, Riccardi C (2015) Note: background oriented Schlieren as a diagnostics for airflow control by plasma actuators. Rev Sci Instrum 86(2):26103

36. Raffel M (2015) Background-oriented schlieren (BOS) techniques. Exp Fluids 56(3):60

37. Velten A, Wu D, Jarabo A, Masia B, Barsi C, Joshi C et al (2013) Femto-photography: capturing and visualizing the propagation of light. ACM Trans Graph 32(4):44

38. Jambunathan K, Lai E, Moss MA, Button BL (1992) A review of heat-transfer data for single circular jet impingement. Int J Heat Fluid Flow 13(2):106-115

39. Lu X, Naidis GV, Laroussi M, Reuter S, Graves DB, Ostrikov K (2016) Reactive species in non-equilibrium atmospheric-pressure plasmas: generation, transport, and biological effects. Phys Rep 630:1-84

40. Kelly S, Golda J, Turner MM, Schulz-Von Der Gathen V (2015) Gas and heat dynamics of a micro-scaled atmospheric pressure plasma reference jet. J Phys D Appl Phys 48(44):444002

41. Jiang N, Yang J, He F, Cao Z (2011) Interplay of discharge and gas flow in atmospheric pressure plasma jets. J Appl Phys 109(9):93305

42. Ghasemi M, Olszewski P, Bradley JW, Walsh JL (2013) Interaction of multiple plasma plumes in an atmospheric pressure plasma jet array. J Phys D Appl Phys 46(5):52001

43. Jiang N, Yang J, He F, Cao Z (2011) Investigation of helium atmospheric pressure plasma jet by Schlieren visualization. IEEE Trans Plasma Sci 39(11):2284-2285

44. Papadopoulos PK, Vafeas P, Svarnas P, Gazeli K, Hatzikonstantinou PM, Gkelios A et al (2014) Interpretation of the gas flow field modification induced by guided streamer ("plasma bullet") propagation. J Phys D Appl Phys 47(42):425203

45. Pei X, Ghasemi M, Xu H, Hasnain Q, Wu S, Tu Y et al (2016) Dynamics of the gas flow turbulent front in atmospheric pressure plasma jets. Plasma Sources Sci Technol 25(3):35013

46. Schmidt-Bleker A, Reuter S, Weltmann K-DD (2015) Quantitative Schlieren diagnostics for the determination of ambient species density, gas temperature and calorimetric power of cold atmospheric plasma jets. J Phys D Appl Phys 48(17):175202

47. Shao X, Chang Z, Mu H, Liao W-L, Zhang G-J (2013) Experimental and numerical investigation on the interaction between Ar Flow Channel and Ar plasma jet at atmospheric pressure. IEEE Trans Plasma Sci. 41(4):899-906

48. Boselli M, Colombo V, Ghedini E, Gherardi M, Laurita R, Liguori A et al (2014) Schlieren high-speed imaging of a nanosecond pulsed atmospheric pressure non-equilibrium plasma jet. Plasma Chem Plasma Process 34(4):853-869 
49. Bradley JW, Oh JS, Olabanji OT, Hale C, Mariani R, Kontis K (2011) Schlieren photography of the outflow from a plasma jet. IEEE Trans Plasma Sci. 39(11):2312-2313

50. Olabanji OT, Bradley JW (2012) Side-on surface modification of polystyrene with an atmospheric pressure microplasma jet. Plasma Process Polym 9(9):929-936

51. Oh J-SS, Olabanji OT, Hale C, Mariani R, Kontis K, Bradley JW (2011) Imaging gas and plasma interactions in the surface-chemical modification of polymers using micro-plasma jets. J Phys D Appl Phys 44(15):155206

52. Foletto M, Puech V, Fontane J, Joly L, Pitchford LC (2014) Evidence of the influence of plasma jets on a helium flow into open air. IEEE Trans Plasma Sci. 42(10):2436-2437

53. Robert E, Sarron V, Darny T, Riès D, Dozias S, Fontane J, Joly L, Pouvesle JM (2014) Rare gas flow structuration in plasma jet experiments. Plasma Sources Sci Technol 23(1):12003

54. Boselli M, Colombo V, Gherardi M, Laurita R, Liguori A, Sanibondi P et al (2015) Characterization of a cold atmospheric pressure plasma jet device driven by nanosecond voltage pulses. IEEE Trans Plasma Sci. 43(3): 713-725

55. Shimizu T, Iwafuchi Y, Morfill GE, Sato T (2011) Formation of thermal flow fields and chemical transport in air and water by atmospheric plasma. New J Phys 13(5):53025

56. Whalley RD, Walsh $J \mathrm{~L}$ (2016) Turbulent jet flow generated downstream of a low temperature dielectric barrier atmospheric pressure plasma device. Sci Rep 6:31756

57. Darny T, Pouvesle JM, Fontane J, Joly L, Dozias S, Robert E (2017) Plasma action on helium flow in cold atmospheric pressure plasma jet experiments. Plasma Sources Sci Technol 26(10):105001

58. Seepersad Y, Pekker M, Shneider MN, Dobrynin D, Fridman A (2013) On the electrostrictive mechanism of nanosecond-pulsed breakdown in liquid phase. J Phys D Appl Phys 46(16):162001

59. An W, Baumung K, Bluhm H (2007) Underwater streamer propagation analyzed from detailed measurements of pressure release. J Appl Phys 101(5):53302

60. Bayoda KD, Benard N, Moreau E (2015) Elongating the area of plasma/fluid interaction of surface nanosecond pulsed discharges. J Electrost 74:79-84

61. Cristofolini A, Neretti G, Roveda F, Borghi CA (2012) Schlieren imaging in a dielectric barrier discharge actuator for airflow control. J Appl Phys 111(3):33302

62. Peschke P, Goekce S, Leyland P, Ott P, Hollenstein C (2013) Experimental investigation of pulsed dielectric barrier discharge actuators in sub- and transonic flow. In: 44th AIAA plasmadynamics and lasers conference, p 2885

63. Peschke P, Goekce S, Leyland P, Ott P (2016) Investigation of nanosecond pulse dielectric barrier discharges in still air and in transonic flow by optical methods. J Phys D Appl Phys 49(2):25204

64. Correale G, Michelis T, Ragni D, Kotsonis M, Scarano F (2014) Nanosecond-pulsed plasma actuation in quiescent air and laminar boundary layer. J Phys D Appl Phys 47(10):105201

65. Erfani R, Zare-Behtash H, Kontis K (2012) Influence of shock wave propagation on dielectric barrier discharge plasma actuator performance. J Phys D Appl Phys 45(22):225201

66. Abe T, Takagaki M (2009) Momentum coupling and flow induction in a DBD plasma actuator. In: AIAA 40th Plasmadynamics and lasers conference, p 4068

67. Gao G, Dong L, Peng K, Wei W, Li C, Wu G (2017) Comparison of the surface dielectric barrier discharge characteristics under different electrode gaps. Phys Plasmas. 24(1):13510

68. Wu B, Gao C, Liu F, Xiong J (2016) Simulation of body force effect on steady and unsteady flow induced by DBD plasma actuator. In: 47th AIAA Plasmadynamics and lasers conference, p 4015

69. Hai D, Zhiwei S, Keming C, Ganniu L, Jichun L, Zheng L et al (2016) The study of flow separation control by a nanosecond pulse discharge actuator. Exp Therm Fluid Sci 74:110-121

70. Belinger A, Hardy P, Gherardi N, Naude N, Cambronne JP, Caruana D (2011) Influence of the spark discharge size on a plasma synthetic jet actuator. IEEE Trans Plasma Sci. 39(11):2334-2335

71. Hardy P, Barricau P, Caruana D, Gleyzes C, Belinger A, Cambronne JP (2010) Plasma synthetic jet for flow control. In: AIAA 40th fluid dynamics conference and exhibit, p 5103

72. Reedy TM, Kale NV, Dutton JC, Elliott GS (2013) Todd reedy Nachiket kale CDGE. Experimental characterization of a pulsed plasma jet. AIAA J 51(8):2027-2031

73. Sosa R, Arnaud E, Mémin E, Artana G (2006) Schlieren image velocimetry applied to EHD flows. In: Proc of the Int symposium on Electrohydrodynamics (ISEHD), pp 331-334

74. Sosa R, Arnaud E, Memin E, Artana G (2009) Study of the flow induced by a sliding discharge. IEEE Trans Dielectr Electr Insul 16(2):305-311

75. Qi XH, Yan HJ, Yang L, Hua Y, Ren CS (2017) Experimental investigation of SDBD plasma actuator driven by AC high voltage with a superimposed positive pulse bias voltage. Phys Plasmas 24(8):83502

76. Feng L, Gao C, Lv Z, Wu B, Zhang Y (2017) Research on the flow characteristics of DBD plasma actuation based on high speed Schlieren. In: 47th AIAA fluid dynamics conference, p 4306

77. Leonov SB, Petrishchev V, Adamovich IV (2014) Dynamics of energy coupling and thermalization in barrier discharges over dielectric and weakly conducting surfaces on $\mu$ s to ms time scales. J Phys D Appl Phys 47(46):465201

78. Takashima K, Zuzeek Y, Lempert WR, Adamovich IV (2011) Characterization of a surface dielectric barrier discharge plasma sustained by repetitive nanosecond pulses. Plasma Sources Sci Technol 20(5):55009

79. Adamovich IV, Little J, Nishihara M, Takashima K, Samimy M (2012) Nanosecond pulse surface discharges for high-speed flow control. In: 6th AIAA flow control conference, p 3137

80. Little J, Takashima K, Nishihara M, Adamovich I, Samimy M (2012) Separation control with nanosecond-pulsedriven dielectric barrier discharge plasma actuators. AIAA J 50(2):350-365

81. Komuro A, Takashima K, Konno K, Tanaka N, Nonomura T, Kaneko T et al (2017) Schlieren visualization of flow-field modification over an airfoil by near-surface gas-density perturbations generated by a nanosecond-pulse-driven plasma actuator. J Phys D Appl Phys 50(21):215202

82. Narayanaswamy V, Clemens NT, Raja LL (2010) Investigation of a pulsed-plasma jet for shock / boundary layer control. In: 48th AIAA aerospace sciences meeting including the new horizons forum and aerospace exposition, p 1089 
83. Opaits DF, Likhanskii AV, Neretti G, Zaidi S, Shneider MN, Miles RB et al (2008) Experimental investigation of dielectric barrier discharge plasma actuators driven by repetitive high-voltage nanosecond pulses with dc or low frequency sinusoidal bias. J Appl Phys 104(4):43304

84. Di J, Yinghong L, Min J, Huimin S, Wei C, Quan S et al (2013) Experimental characterization of the plasma synthetic jet actuator. Plasma Sci Technol 15(10):1034

85. Pavon S, Ott P, Leyland P, Dorier J-L, Hollenstein C (2009) Effects of a surface dielectric barrier discharge on transonic flows around an airfoil. In: 47th AIAA aerospace sciences meeting including the new horizons forum and aerospace exposition, p 649

86. Popov IB, Nikipelov A, Pancheshnyi S, Correale G, Hulshoff SJ, Veldhuis LLM et al (2013) Experimental study and numerical simulation of flow separation control with pulsed nanosecond discharge actuator. In: 51st AIAA aerospace sciences meeting including the new horizons forum and aerospace exposition, p 572

87. Fomin VM, Malmuth N, Maslov AA, Fomichev VP, Shiplyuk AN, Pozdnyakov GA et al (1999) Influence of a counter plasma jet on the integral and distributed aerodynamic characteristics of a blunt body. In: First workshop on MHD and plasma technology in aerospace applications, Moscow, IVTAN, pp 72-74

88. Cybyk BZ, Grossman KR, Wilkerson JT, Chen J, Katz J (2005) Single-pulse performance of the sparkjet flow control actuator. In: 43rd AIAA aerospace sciences meeting and exhibit, p 401

89. Moreau E, Sosa R, Artana G (2008) Electric wind produced by surface plasma actuators: a new dielectric barrier discharge based on a three-electrode geometry. J Phys D Appl Phys 41(11):115204

90. Arnaud E, Mémin E, Sosa R, Artana G (2006) A fluid motion estimator for Schlieren image velocimetry. In: Computer vision - ECCV 2006, Pt 1, proceedings, vol 3951, pp 198-210

91. Lindsey WF. Device for evaluating the sensitivity of an optical system utilizing the Foucault knife edge test. US patent 2777355, 1957

92. Colombo V, Concetti A, Ghedini E, Dallavalle S, Vancini M (2009) High-speed imaging in plasma arc cutting: a review and new developments. Plasma Sources Sci Technol 18(2):23001

93. Siewert E, Wilhelm G, Haessler M, Schein J, Hanson T, Schnick M et al (2014) Visualization of gas flows in welding arcs by the Schlieren measuring technique. Weld J 93(1):1S-5S

94. Moen WB, Gibson GJ (1952) Schlieren analysis of inert-gas arc shields. Weld J 31(3):208-213

95. Pfender E, Fincke J, Spores R (1991) Entrainment of cold gas into thermal plasma jets. Plasma Chem Plasma Process 11(4):529-543

96. Fincke JR, Swank WD, Haggard DC (1993) Entrainment and demixing in subsonic argon / helium thermal plasma jets. J Therm Spray Technol 2(4):345-350

97. Russ S, Strykowski PJ, Pfender E (1994) Mixing in plasma and low density jets. Exp Fluids 16(5):297-307

98. Raghu S, Goutevenier G, Gansert R (1995) Comparative study of the structure of gas-stabilized and water-stabilized plasma jets. J Therm Spray Technol 4(2):175-178

99. Henne RH, Bouyer E, Borck V, Schiller G (2001) Influence of anode nozzle and external torch contour on the quality of the atmospheric DC plasma spray process. Therm Spray 2001 New Surfaces a New Millenn 1:471-478

100. Hurba O, Hlina M, Hrabosky M (2016) Diagnostic of plasma jet generated in water/argon DC arc torch. Plasma. Phys Technol 3(1):5-8

101. Nagulin KY, Akhmetshin DS, Gilmutdinov AK, Ibragimov RA (2015) Three-dimensional modeling and Schlieren visualization of pure Ar plasma flow in inductively coupled plasma torches. J Anal At Spectrom R Soc Chem 30(2):360-367

102. Boselli M, Cavrini F, Colombo V, Ghedini E, Gherardi M, Laurita R et al (2014) High-speed and Schlieren imaging of a low power inductively coupled plasma source for potential biomedical applications. IEEE Trans Plasma Sci. 42(10):2748-2749

103. Barbieri D, Boselli M, Cavrini F, Colombo V, Gherardi M, Landini MP et al (2015) Investigation of the antimicrobial activity at safe levels for eukaryotic cells of a low power atmospheric pressure inductively coupled plasma source. Biointerphases 10(2):29519

104. Traldi E, Boselli M, Gherardi M, Colombo V (2017) A simulative and experimental approach for the design and optimization of atmospheric pressure low power RF thermal plasma processes. Plasma Process Polym 14(4-5):1600167

105. Bemis BL, Settles GS (1998) Visualization of liquid metal, arc, and jet interactions in plasma cutting of steel sheet. In: 8th international symposium on flow visualization (Sorrento)

106. Kim S, Heberlein J, Lindsay J, Peters J (2010) Methods to evaluate arc stability in plasma arc cutting torches. J Phys D Appl Phys 43(50):505202

107. Kim S, Heberlein J, Lindsay J, Peters J (2012) Torch design modification using micro-jets to suppress fluid dynamic instabilities in plasma arc cutting. Plasma Chem Plasma Process 32(1):45-63

108. Cantoro G, Colombo V, Concetti A, Ghedini E, Sanibondi P, Zinzani F (2011) Statistical analysis of high-speed Schlieren imaging in PAC. IEEE Trans Plasma Sci. 39(11):2898-2899

109. Prevosto L, Artana G, Kelly H, Mancinelli B (2011) Departures from local thermodynamic equilibrium in cutting arc plasmas derived from electron and gas density measurements using a two-wavelength quantitative Schlieren technique. J Appl Phys 109(6):63302

110. Kiyohara M, Okada T, Yamamoto H (1977) Observation of the shielding gas flow pattern during arcing by the use of a laser light source. J Japan Weld Soc 46(1):1011-1016

111. Schnick M, Dreher M, Zschetzsche J, Füssel U, Spille-Kohoff A (2012) Visualization and optimization of shielding gas flows in arc welding. Weld World 56(1-2):54-61

112. Bitharas I, Campbell SW, Galloway AM, MCPherson NA, Moore AJ (2016) Visualisation of alternating shielding gas flow in GTAW. Mater Des 91:424-431 